\title{
La sustentabilidad en las instituciones de Educación Superior
}

Sustainability in Higher Education Institutions

\author{
Irma I. de León-Vázquez a , Filiberta de la Peña-de la Cruz ${ }^{b}$, Shadai B. Escamilla-Mendoza ${ }^{c}$,
} Delia I. Tapia-Castillo ${ }^{d}$ Daniel Vélez-Díaz ${ }^{e}$

\begin{abstract}
:
Reflective work on the importance of sustainability in higher education institutions and find the problem that can not be used as such, change the ideologies of others is difficult, however, the situation of our environment is not taken into account. It shows the context, and the generalized environmental degradation that transcends the ecological field and puts in check the development of society; It may be asked if there is a responsibility on the part of the universities to teach, train and research on sustainability issues. Several concepts related to the subject are researched and it is considered that the university students must become aware and the teachers and institutions support the act of sustainability.
\end{abstract}

\section{Keywords:}

Sustainability, Ideology, Environmental degradation.

\section{Resumen:}

El trabajo reflexiona sobre la importancia de la sustentabilidad en las instituciones de educación superior y encontrar la problemática de que no se lleve como tal, cambiar la ideologías de los demás es difícil, sin embargo no está por demás tomar conciencia de la situación de nuestro alrededor. Muestra el contexto, y la generalizada degradación ambiental que trasciende el ámbito ecológico y pone en jaque el desarrollo de la sociedad; cabe preguntarse si existe una responsabilidad por parte de las universidades en enseñar, capacitar e investigar sobre temas de sostenibilidad. Se investigaron varios conceptos relacionados con el tema y se considera que los universitarios deben tomar conciencia y que los docentes e instituciones apoyen al acto de sustentabilidad.

\section{Palabras Clave:}

Sustentabilidad, Ideología, Degradación ambiental.

\section{Introducción}

La sustentabilidad es un término ligado a la acción del hombre en relación a su entorno. Dentro de la disciplina ecológica, la sustentabilidad se refiere a los sistemas biológicos que pueden conservar la diversidad y la productividad a lo largo del tiempo. Sin embargo, también se ha ligado al equilibrio de cualquier especie en particular considerando los recursos con los que se encuentran en su entorno. En 1987, se realizó el Informe Brundtland, dentro de la acción de Naciones Unidas, y que la definió como "la capacidad de satisfacer

\footnotetext{
a Autor de Correspondencia, Profesor por asignatura de la Universidad Autónoma del Estado de Hidalgo, Escuela Superior de Tlahuelilpan. E-mail: irmalv@uaeh.edu.mx

b Alumna de la Universidad Autónoma del Estado de Hidalgo, Escuela Superior de Tlahuelilpan. E-mail: fili_324@ hotmail.com

c Alumna de la Universidad Autónoma del Estado de Hidalgo, Escuela Superior de Tlahuelilpan. E-mail: shadai_sol@hotmail.com

d Profesor de Tiempo completo de la Universidad Autónoma del Estado de Hidalgo, Escuela Superior de Tlahuelilpan. E-mail: itapia@uaeh.edu.mx

${ }^{\text {e }}$ Profesor Investigador de la Universidad Autónoma del Estado de Hidalgo, Escuela Superior de Tlahuelilpan. E-mail: 
necesidades de la generación humana actual sin que esto suponga la anulación de que las generaciones futuras también puedan satisfacer sus necesidades propias". La constante recuperación ambiental del globo terráqueo, y en especial de México, exige la participación positiva de todos los seres humanos. Esta tarea es urgente y requiere de una gran organización y de compromisos expresos que reflejen auténtica voluntad transformadora, como puede ser la acción que atañe a los mexicanos de hoy en beneficio de sus hijos y de sus descendientes, por tal razón, es apremiante diseñar medidas y acciones que ayuden a minimizar o salvar los impactos negativos al medio ambiente, y a su vez contribuir a la renovación y el equilibrio de los mismos. La afirmación de que no puede haber desarrollo económico y social a largo plazo en un planeta devastado, y que la tarea de enseñar a la sociedad a comportarse de manera responsable y a respetar el entorno, es obligación de ser humano.

Ante un escenario mundial desafiante y dinámico, las grandes organizaciones han asumido responsabilidades en materia de medio ambiente y desarrollo sostenible, que deberían de animar la educación superior a los alumnos para hacer mejor conciencia, ahora bien, si se toma a la Universidad como el epítome de la Institución de Educación Superior, es recomendable recordar que la palabra "Universidad" tiene su origen en el Latín "universitas magistrorum et scholarium," que podría traducirse como "comunidad de profesores y escolares". Dedicada a la especialización, al entrenamiento y a la concesión de títulos.

Ahora bien, las instituciones de educación superior se han percibido - históricamente - como semillero de ideas, sitio de creación y libre pensamiento. Sin embargo, en lo que corresponde a la intervención en el desarrollo sustentable se distingue en tres ámbitos.

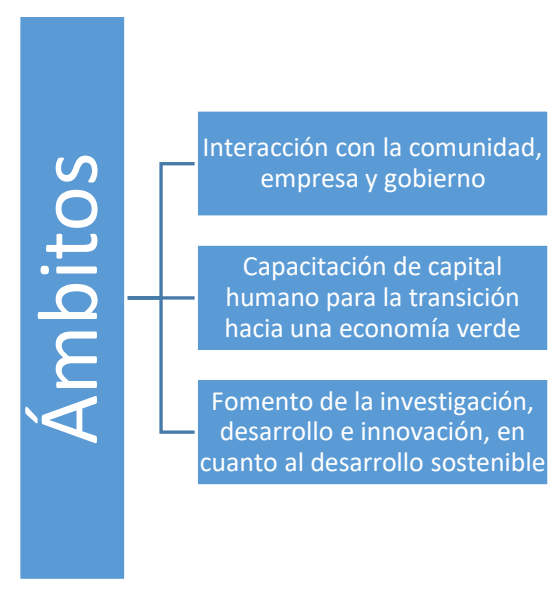

Figura 1. Ámbitos en el desarrollo sustentable de las Universidades
La interacción con el entorno es vital para empujar a la sociedad por la ruta sustentable, es el espíritu de abstracción del ambiente universitario; la interacción con el entorno es casi inexistente, dejando en el cajón estudios que podrían tener un impacto positivo real en la región que habita y rodea a la propia Universidad, un ejemplo puede considerarse desde la caracterización de procesos industriales para la mejora del eco desempeño de industrias hasta la difusión de un estudio que ayude al rediseño de rutas de transporte público.

La capacitación es intrínseca a una institución formadora de ideas noveles y de recursos humanos de alto nivel que en tiempo serán tomadores de decisiones. La capacitación también puede ser parte de la interacción con el sector privado/productivo y el gubernamental.

Finalmente, el fomento a la investigación tiene un nicho en las universidades, mas debe ir a acompañada de apoyo gubernamental y del sector público y privado. $\mathrm{Y}$ a este punto es necesario visitar algunas cifras, si consideramos como indicador al porcentaje del PIB que se destina a todo el sector de educación (básica, media y superior) se tiene que en 2009, fue $5.7 \%$ del PIB. Los objetivos en general es Impulsar el fortalecimiento y compromisos responsable y permanente de los alumnos de educación superior, enfocados en tres áreas fundamentales en el cuidado del medio ambiente: Primero como el desarrollo sostenible asumir los postulados y principios mundiales a favor del medio ambiente y el desarrollo sostenible, segundo hacer conciencia ambiental activa al lograr la participación de los alumnos en el cuidado y manejo responsable de los recursos naturales, creando una conciencia ambiental activa a fin de lograr un efecto multiplicador que permee entre alumnos y familias y tercero inmuebles sustentables acreditar los activos de la infraestructura "inmuebles sustentables", propiciando las acciones necesarias que aseguren el funcionamiento respetuoso del ecosistema y vigilar que apliquen las políticas de sostenibilidad en el uso de energía eléctrica, agua, ventilación y tratamiento de desechos.

\section{Desarrollo}

Sustentabilidad se ha definido como la cualidad de sustentable. Para las áreas como la economía y la ecología, sustentable es algo que se puede sostener a lo largo del tiempo sin agotar sus recursos 0 perjudicar el medio ambiente.

En este sentido, la sustentabilidad es la capacidad que tiene una sociedad para hacer un uso consciente y responsable de sus recursos, sin agotarlos o exceder su capacidad de renovación, y sin comprometer el acceso a estos por parte de las generaciones futuras. 
La misión que la sociedad ha conferido a las Instituciones de Educación Superior es la de "transmisión, generación, aplicación y difusión del conocimiento científico, tecnológico y humanístico". Por ello, las políticas generales y mecanismos de trabajo, así como las líneas de acción, deben reconocer esta responsabilidad fundamental de manera concordante con el perfil y las características institucionales, y buscar reforzar la contribución al desarrollo, esto es que:

1. Se reconozca que los problemas del desarrollo sustentable son altamente complejos y, por lo tanto, requieren del concurso de las diferentes profesiones y disciplinas, en una labor académica del más alto nivel de calidad, pertinencia y equidad.

2. Dada la magnitud y amplitud del tema del desarrollo sustentable es necesario construir marcos de acción que visualicen la totalidad de la problemática, y que por lo tanto impulsen el desarrollo de acciones para la formación en educación ambiental que contribuya a que todo egresado de la educación superior comprenda, fomente y difunda una cultura ambiental orientada a disminuir los riesgos al ambiente y garantizar así un desarrollo armónico, equilibrado, y con posibilidades de sustentabilidad en el tiempo.

3. Los programas y proyectos sean el resultado, en primer lugar, de la colaboración interinstitucional, con énfasis en el trabajo horizontal, llevado a cabo por diferentes dependencias académicas, y en segundo lugar, de la cooperación con otras instituciones educativas del nivel superior y de otros niveles educativos, así como con los organismos gubernamentales, privados y de la sociedad civil.

4. Se dé una mayor difusión a los resultados de investigaciones y programas educativos y de extensión en educación ambiental y desarrollo sustentable a fin de compartir los conocimientos y facilitar su utilización en las diferentes regiones del país.

La sustentabilidad en las instituciones de educación superior está tocando aspectos que no fueron imaginados en 1987, cuando se hablaba de tomar en cuenta los límites ambientales. La sustentabilidad es un cuerpo geométrico que puede ser descrito por diversas dimensiones, las de mayor tradición han sido la social, la económica y la ambiental. Pero hoy en día, diferentes autores dependiendo de su disciplina y sector hablan de otras dimensiones, por ejemplo: la académica, espiritual, arquitectónica, política, paisajística, mismas que cobran importancia en función del contexto que se tiene, y el rumbo que se le quiere dar a la sustentabilidad.

\section{La Educación ambiental y su objeto}

La Educación Ambiental ha sido definida como "el proceso de adquisición de valores y clarificación de conceptos cuyo objetivo es desarrollar actitudes y capacidades necesarias para entender y apreciar las interrelaciones entre el hombre, su cultura y su entorno biofísico". La educación ambiental también incluye la formación de la persona para que participe en la toma de decisiones y la formulación de un código de conducta relacionado con los temas relativos a la calidad ambiental de manera ideal, la educación ambiental es un proceso por el cual las personas llegan a cuestionar su relación con el ambiente, y a comprender el impacto que sus acciones tienen en los sistemas naturales que los rodean. El propósito fundamental de la educación ambiental es generar una sensibilización hacia la necesidad de cuidar el medio ambiente. No obstante, la educación ambiental debe también modificar actitudes y proporcionar nuevos conocimientos y criterios más allá de los conceptos puramente ecológicos. La educación ambiental debe contemplar al individuo desde una perspectiva ecológica, como un ser integrante de un ecosistema, que sitúa al hombre como dominador de la naturaleza, para favorecer una relación de éste con el medio ambiente basada en el respeto de los ciclos naturales. Donde la conducta de uso correcto de los recursos del planeta viene a constituirse en uno de los objetivos finales del proceso educativo. De acuerdo con esto, surge la necesidad de tener un mejor conocimiento del medio ambiente, de adquirir habilidades apropiadas para utilizar este conocimiento, y de iniciar acciones tendientes a un uso sensato del medio ambiente sin perturbar el equilibrio ecológico.

La Conferencia Intergubernamental sobre Educación Ambiental, organizada por UNESCO en colaboración con el PNUMA, en octubre de 1977 en Tbilisi, aprobó los siguientes propósitos, objetivos y principios orientadores para la Educación Ambiental. 


\section{Propósitos o metas}

- Promover una clara conciencia e interés por la interdependencia económica, social, política y ecológica en los sectores urbanos y rurales.

- Proporcionar a cada persona las oportunidades para adquirir los conocimientos, valores, actitudes, compromisos y habilidades para proteger y mejorar el medio ambiente.

- Crear nuevos patrones de conducta hacia el medio ambiente para los individuos, grupos y la sociedad.

\section{Objetivos (ayudar a los grupos sociales e} individuos)

- Adquirir una conciencia de sensibilidad hacia el ambiente y sus problemas asociados.

- Tener una serie de experiencias y adquirir un conocimiento básico en relación con el medio ambiente y sus problemas conexos.

- Adquirir una serie de valores y de sentimientos de preocupación por el medio ambiente, así como la motivación para participar activamente en el mejoramiento y protección ambientales.

- Adquirir las habilidades necesarias para identificar y resolver problemas ambientales (Habilidades).

- Tener una oportunidad para comprometerse activamente y a todo nivel en el trabajo a favor de la resolución de los problemas ambientales.

Figura 2. Propósitos y objetivos orientados a la Educación Ambiental

\section{Educación Ambiental a Nivel Superior}

Son numerosos los pronunciamientos que manifiestan la necesidad de la educación ambiental a nivel superior.
En el informe final de la Conferencia de Tbilisi, referente a las universidades, se establece que:

\begin{abstract}
"Las universidades, en su calidad de centros de investigación, de enseñanza y de formación del personal calificado del país, deben dar cada vez mayor cabida a la investigación sobre Educación Ambiental y la formación de expertos en educación formal y no formal. La Educación Ambiental es necesaria para los estudiantes, cualquiera que sea la disciplina que estudien, ya sean las Ciencias Exactas y Naturales, las Ciencias Sociales o las Artes, puesto que la relación que guardan entre sí la naturaleza, la técnica y la sociedad marca y determina el desarrollo de una sociedad".
\end{abstract}

El Programa de las Naciones Unidas para el Medio Ambiente (1985) reconoce la importancia de la educación y de la investigación ambiental, ya que a las Instituciones de Educación Superior (IES) corresponde el papel relevante en la generación y transmisión del conocimiento.

Por su parte, en el Congreso Iberoamericano de Educación Ambiental (1992) se expuso la necesidad de integrar la problemática ambiental al currículo de las carreras universitarias. Los expertos ahí reunidos coincidieron en que el diseño de programas de estudio a nivel licenciatura contribuirá a reforzar los valores del estudiante, dándole una formación en materia ambiental. Las IES tienen hoy grandes retos en materia ambiental como son: la generación de programas de educación ambiental para la transmisión de conocimientos, la conformación de grupos académicos capaces de abordar y plantear los problemas que requieren de la participación articulada de diversas disciplinas.

\section{Acciones Necesarias}

Las IES deben realizar investigación y producir tecnología que favorezca el desarrollo sostenible del país. Por ello, el conocimiento de los factores ambientales y sus relaciones reciprocas son básicos, por lo que deben apoyarse proyectos que generen conocimiento $y$ contribuyan a solucionar o disminuir los problemas ambientales locales, nacionales y mundiales; proyectos técnicos que controlen o eliminen los niveles de contaminación, que construyan tecnologías limpias de producción, o permitan una mejor explotación de los recursos naturales. "Es necesario incorporar la perspectiva ambiental en todos los currículos de licenciatura y posgrado que ofrecen las IES así como los programas de capacitación, actualización profesional o educación continua de manera que proporcionen una formación ambiental, humana y social integral que 
permita a sus egresados tomar decisiones como adultos responsables y como profesionistas competentes". Se recomienda que las IES desarrollen procesos de formación integral en las diversas áreas del conocimiento para la "detección, prevención y manejo profesional de los problemas del medio ambiente". El desarrollo sostenible requiere de una estrategia educativa que tienda a modificar los actuales estilos de vida, los parámetros consumistas de la civilización, los valores imperantes que, en definitiva, hacen que lo verdaderamente insostenibles sean nuestras vidas. Es más, la educación es un instrumento adecuado para el cambio no sólo individual sino también social; no existe duda que la suma de personas educadas en unos mismos valores hacen posible a la larga, cambios comunitarios y sociales. Por otra parte, es necesario que existan más personas que acepten y valoren la necesidad de la sostenibilidad y del desarrollo. Se debe buscar un cambio educativo y un compromiso radical que modifique las premisas axiológicas del crecimiento e inicie la travesía que nos lleve del tener al ser. Sólo en estas condiciones, se podrá estar y perdurar en el planeta. La Universidad como entidad docente e investigadora es el principal agente de cambio que debe proporcionar respuestas a los problemas de la sociedad: tiene que alumbrar nuevos paradigmas que expliquen la realidad; experimentar científica y tecnológicamente las soluciones a dichos problemas y capacitar al capital humano que debe emprender el cambio. Cada vez son más numerosas las universidades que incorporaron la variable ambiental en su docencia e investigación, en su gestión $y$, en general, en la vida universitaria. Comprometerse con el deterioro ambiental e iniciar actuaciones que se relacionan con la gestión de residuos o la educación ambiental es una opción ética de cada institución universitaria, que pretende con ellas convertirse en un centro modélico de desarrollo ambientalmente sostenible A continuación se agrupan las actuaciones de gestión ambiental que se puede encontrar en los centros universitarios, si bien la problemática particular de cada universidad hará que sea más importante trabajar en unos u otros temas:

1- Gestión y minimización de residuos: cuando se trata de residuos peligrosos, más importante, porque se relaciona con el cumplimiento de la legislación en materia de prevención de riesgos laborales.

2- Gestión del agua: en un entorno como el mediterráneo, los recursos hídricos constituyen una pieza fundamental de la sostenibilidad, desde su uso, y el necesario ahorro, hasta la evacuación y tratamiento de las aguas residuales.
3- Gestión energética: el consumo energético será mayor en universidades politécnicas 0 de ciencias aplicadas. También, el facto educativo en el ahorro energético en la vida universitaria es importante.

4- Gestión de la movilidad y el transporte: Las universidades deben fomentar la utilización de modos de transporte sostenible: dentro de su institución por medio de vehículos eléctricos o favoreciendo un diseño para el paseo o la utilización de la bicicleta; también demandando a los organismos competentes un transporte público (tren, autobús, metro) de calidad, y que cumpla con un servicio que un gran público cautivo utiliza durante más de nueve meses al año. En relación a los vehículos privados, intentar desincentivar su uso (ejemplo, cobro por el aparcamiento) pueden ser actuaciones que en la actualidad resulten extremas, pero que a corto plazo se puedan concretar por el aumento de vehículos que se podrán registrar en las universidades.

5- Diseño, ordenación urbana y construcción sostenible: este aspecto es importante en la medida en que es estructural. Algunos ejemplos: de la distancia y accesibilidad desde zonas urbanas a la universidad en campus externos, dependerá la utilización de medios de transporte; del diseño de zonas ajardinadas dependerá el consumo de agua; del diseño arquitectónico dependerá el consumo energético de los edificios y de su situación en el campus y/o en la ciudad, el impacto acústico, si está cerca de infraestructuras de transporte y no está aislado convenientemente.

6- Compra verde y criterios ambientales en contrataciones: la gestión ambiental de la universidad también depende de la gestión de las empresas que le prestan servicios o les proporcionan bienes. Las universidades tienen que apostar por adquirir bienes que cumplan con requisitos ambientales (por ejemplo, maderas certificadas, papel reciclado o libre de cloro, equipos informáticos reutilizables y duraderos, etc.) y seleccionar servicios cuyas empresas tengan certificaciones en sistemas de gestión ambiental. Los servicios de cafetería, jardinería, limpieza, reprografía; las empresas gestoras de los equipamientos deportivos deben hacer una gestión coherente con la que haga la universidad en las que trabajan: comprometida con la sostenibilidad. A la hora de planificar las acciones de intervención en la gestión se debe procurar incorporar también la componente 
educativa. Estas intervenciones tendrán en forma indirecta un efecto en cascada que hace que las personas concienciadas puedan transferir dichos modelos de gestión a otros ámbitos 0 incluso incorporarlos a las instituciones públicas o privadas en las que pueda desarrollar en el futuro su profesión.

\section{La educación para la sostenibilidad en la universidad}

Una de las funciones principales de la universidad, es la educación de los estudiantes. En cuanto a educación ambiental se refiere, se puede distinguir dos grupos de actuaciones bien definidas:

1. Una educación ambiental universitaria formal, en la medida en que forma parte de la estructura y organización convencional de la educación universitaria. Por educación ambiental universitaria se entiende aquella que trata de integrar el medio ambiente y la sostenibilidad en la academia, es decir, en la docencia y en la investigación.

2. Una educación ambiental universitaria no formal, parte de la gestión ambiental que una universidad emprende. Se incluyen actividades de educación y participación ambiental, para aumentar la sensibilización y concienciación; además, promover conductas pro-ambientales de toda la comunidad universitaria a través de charlas, conferencias, jornadas, exposiciones, talleres, etc. que se refieran a temas ambientales de actualidad, con relación o no al medio ambiente universitario. Las actividades extracurriculares desempeñan un rol significativo en el desarrollo de la personalidad del universitario, permiten conectar a la universidad y a los estudiantes de forma directa con los problemas y sus soluciones de la actualidad ambiental.

\section{Las políticas para la sustentabilidad de las IES en México:}

En un análisis comparativo de la estructura y los pronunciamientos de los 35 planes institucionales que tiene registrados la ANUIS más los 18 que se encuentran inscritos en el Consorcio Mexicano de Programas Ambientales Universitarios para el Desarrollo Sustentable (COMPLEXUS), se reconoce una serie de elementos en común que son clave para la comprensión de las dificultades que encierran las políticas para la sustentabilidad desde su diseño.
Marco y antecedentes de las políticas. Todos los planes, sin excepción, mencionan como marco la dinámica internacional de reuniones, cumbres, estatutos, documentos y acuerdos como antecedente de las políticas para la sustentabilidad; no obstante, los pronunciamientos y disposiciones citados se toman textuales, con escasa relación al contexto y la identidad de cada institución.

Objetivos. Las políticas están dirigidas a insertar el tema ambiental en las funciones sustantivas de cada universidad: docencia, investigación y vinculación (extensión y difusión), con una tendencia al diseño de acciones, programas y proyectos para cada una. La particularidad que presentan los objetivos es una falta de articulación entre las diferentes acciones, aunque esta cualidad de la política para la sustentabilidad puede ser sólo un reflejo de una característica estructural atribuible a las universidades; mientras los planes de estudio privilegian la docencia, la investigación es insuficiente y aislada, la extensión es ocasional y descoordinada, además de que una de otra se encuentra desvinculadas

Estrategias. Las estrategias varían entre los 47 planes, pero se pueden agrupar de la siguiente manera:

Introducir como primer paso la sensibilización, la concientización y la formación ambiental a través de recursos como cursos y talleres.

Establecer proyectos específicos al interior de la institución que versen alrededor de "temas prioritarios o clave” como residuos sólidos, agua y energía.

Diseño de Sistemas de Gestión Ambiental (SGA). En este sentido hay una inclinación por alcanzar la certificación ISO 14001 sobre todo por parte de los institutos tecnológicos.

El enverdecimiento de las instalaciones.

Extender o reproducir la experiencia de las áreas biológicas a toda la institución.

Implementar estrategias de vinculación ideadas hacia la instauración de convenios y proyectos, con el sector gubernamental sobre todo y con el empresarial en segunda instancia.

En general, hay un débil contenido social en las propuestas y en la exposición de las problemáticas 
Existe confianza en la reforma curricular como vía para transversalizar el tema ambiental en la docencia y en el modelo de la educación integral centrada en el estudiante como un peldaño necesario para la integración de dichos temas.

Las estrategias que proponen las IES reflejan, en gran medida, los elementos sugeridos por la ANUIES en su propuesta metodológica, la cual brindaba un guion basado en el modelo de planificación estratégica, aunque muchas de las universidades que promueven los sistemas de gestión ambiental y las estrategias relacionadas con el ahorro y la disminución de la huella ecológica.

Si bien la estrategia promovía la elaboración de un plan de acción para el Desarrollo Sustentable, tuvo una fuerte predominancia el tópico de lo ambiental, al grado de que una gran mayoría fue denominada como Planes Ambientales Institucionales. Ese desequilibrio marcó en gran medida las estrategias, y en el mayor número de casos, una ausencia de temas sobre todo de índole social; esta asimetría no responde a un mero capricho de la institución, sino a un fenómeno que atiende razones teóricas y éticas más de fondo.

Los resultados de la encuesta de la USLF también señalan que el grado de abstracción y la amplitud del significado de la sustentabilidad representan un obstáculo para la implementación de políticas. La sustentabilidad es un campo conflictivo y polisémico, no sólo una noción con múltiples definiciones; es un proceso dinámico que no comulga con la idea de meta ni de perpetuar un status quo. El cambio y la adaptación son justamente las propiedades constitutivas de la sustentabilidad, al igual que los sistemas sometidos a dinámicas evolutivas. La principal discrepancia en estas discusiones versa sobre la elasticidad de los sistemas naturales, donde las relaciones entre hombre y naturaleza exterior se han concebido como una relación meramente técnica, esto es, no están consideradas como parte de las relaciones humanas.

Desde la visión occidental dominante, las personas son fundamentalmente diferentes de todas las demás criaturas a las cuales dominan; se considera que la herencia cultural, además de la genética, hace al ser humano diferente a las otras especies, y si bien el medio ambiente es un determinante de las características de las sociedades humanas, la relación con éste sucede teniendo como intermediario a la tecnología.

Bajo esta óptica, los problemas sociales son incluidos en la sustentabilidad porque implican un deterioro ecológico, y el resultado de ello es la aplicación de las soluciones técnicas o reglamentarias que modifican muy poco las relaciones sociales actuales, mejor dicho, las relaciones capitalistas actuales.

La mayor parte de las posturas sobre la sustentabilidad sólo implican corregir el capitalismo, e incluso hasta en quienes llaman a realizar cambios en el estilo de vida, sus modificaciones se aplican dentro de las mismas relaciones sociales predominantes.

Con esta limitada concepción de la sustentabilidad es como se forjan las políticas que han establecido las universidades. La esencia reproductora de las IES no convive con promover los cambios en las estructuras universitaria y social, aquéllas se han preocupado más por generar conocimiento para remediar los efectos que el sistema económico ha producido, sin considerar prioritario modificar su propia forma de organización.

\section{Resistencias en tiempos de cambio}

La postura "suave" de las políticas para la sustentabilidad en las IES, en el fondo, busca mantener su tradicional estructura, aunque la resistencia no se ubica en la posibilidad del cambio o no, sino en cómo sucederá y cuándo. La Universidad es una institución que desde su nacimiento, aproximadamente en el siglo $x x$, ha vivido cambios esenciales; sin embargo, la forma en que hoy la conocemos aún conserva la herencia del modelo humboldtiano, esto es, los cambios suceden lentamente, se filtran a través de la organización universitaria, en la cual confluyen y se mueven todo tipo de actores que metabolizan y hacen complejo el tránsito hacia nuevos cambios como la interdisciplinariedad, la sustentabilidad, la complejidad, etc.

Si centramos la atención sólo en el abordaje de la interdisciplinariedad como un componente necesario para el abordaje holístico de la sustentabilidad, la variedad de formas que se refiere en los planes deja múltiples vacíos respecto a los mecanismos de interrelación o coordinación efectiva entre las disciplinas. De algún modo, la organización de los campos disciplinarios se mantiene intacta, abriendo sólo algunas vías de comunicación o estableciendo algunos puentes de intercambio como el trabajo en equipo, la formación de academias, la reestructuración curricular con enfoques multidisciplinarios, la elaboración de proyectos y líneas de investigación, o incluso el diseño de algunos programas con intenciones interdisciplinarias que consisten únicamente en aportar elementos pedagógicos que integran elementos de la naturaleza como recurso educativo. 
La interdisciplinariedad no puede ser sólo un enfoque como lo menciona un gran número de planes, pues implica todo un debate cognoscitivo, social y cultural en la reforma de la Universidad, pero tampoco se puede eludir el hecho de que las universidades descansan en la especialización, por lo que la disciplina es considerada como la categoría organizadora del conocimiento científico por excelencia y la columna vertebral de la estructura académica que sostiene a las instituciones, dándoles permanencia y sentido. Cada disciplina tiende a la autonomía y a delimitar sus fronteras favorecidas por el lenguaje, por las técnicas y por las teorías propias.

La vía de la especialización genera cuerpos de conocimiento con escasa relación entre sí y este orden es el que limita la posibilidad de abordar de una manera integral los problemas socio-ambientales de hoy. Sin embargo, integrar el conocimiento es un tema complejo en tanto que sigue siendo imprescindible la especialización como mecanismo para tener un mayor conocimiento del mundo; si este debate lo llevamos al campo universitario, resulta doblemente complejo, en tanto que se tiene, por un lado, la existencia de los imaginarios fronterizos y, por otro, su institucionalización. Las ciencias naturales suelen ser más disciplinarias que las sociales; estas últimas llevan una ventaja en tanto que han propuesto la intervención de equipos de especialistas de múltiples disciplinas para el abordaje de determinados problemas sociales, pero son menos comunes las modalidades interdisciplinarias en las que hay que emplear metodologías propias y de otras áreas de conocimiento o construir marcos teóricos comunes.

Las estrategias interdisciplinarias no pueden consistir únicamente en una declaración de buenas intenciones o en un mero enfoque como medio para las políticas para la sustentabilidad, su falta de claridad puede generar más obstáculos de los que pretenda resolver y la ausencia de instrumentos estratégicos de gestión puede llevar a las IES a reestablecer el aislamiento.

\section{Conclusiones}

La Educación Ambiental debe ayudar a desarrollar una conciencia ética sobre todas las formas de vida con las cuales compartimos este planeta; respetar sus ciclos vitales e imponer límites a la exploración de esas formas de vida por los seres humanos. Las IES juegan un papel de suma importancia en la adquisición de valores y conocimientos, por lo que deben brindar ya una educación ambiental formal y no formal a los estudiantes y a los profesionistas egresados. A pesar de los esfuerzos realizados por la IES a nivel nacional se tiene una insuficiente oferta de programas educativos, tanto formales como no formales que aborden directamente los problemas ambientales. Es necesario dar mayor énfasis a la formación de grupos académicos interesados en la problemática ambiental $\mathrm{y}$, que sustenten los programas educativos, además de un mayor impulso a la investigación interdisciplinaria que permita generar nuevos conocimientos y alternativas viables para la solución de los problemas ambientales. Las IES deberán ser capaces de generar y difundir los conocimientos necesarios para soportar una estrategia que permita avanzar hacia la sustentabilidad y sostenibilidad del desarrollo de acuerdo a los temas desarrollados en esta oportunidad, se llega a la conclusión que urge la necesidad de incorporación en la malla curricular de las diferentes carreras que se desarrollan en las universidades de materias que se relacionan con el medio ambiente de tal forma que se haga efectivo el desarrollo sostenible.

\section{Referencias}

Dorantes García, S. (18 de enero de 2018). DAMIE. Obtenido de DAMIE: http://ww.farma.com

Flores Cortéz, A. (23 de octubre de 2010). GARFE. Obtenido de GARFE: http://www.gusme.com

Gomez Pérez, D. (21 de enero de 2005). Corporation. Obtenido de Corporation: htt://www.renau.com

Hernández Juárez, R. (24 de agosto de 2016). Denis. Obtenido de Denis: http://www.guzman.com

Ordoñes Ortiz, F. (23 de Noviembre de 2015). UNAM. Obtenido de UNAM: http://www.adatum.com

UNAM. (2015). Plan de Desarrollo. México: UNAM.

Universidad Autónoma del Estado de Hidalgo. (2000). La Sustentabilidad en las Universidades. Hidalgo: UAEH. 